\title{
Rapid isolation and identification of pneumonia associated pathogens from sputum samples combining an innovative sample preparation strategy and array-based detection
}

Susanne Pahlow $\$ 1,2$, Lydia Lehniger ${ }^{\ddagger 2,3}$, Stefanie Hentschel ${ }^{2,30}$, Barbara Seise ${ }^{2,3}$, Sascha D. Braun $^{2,4}$, Ralf Ehricht ${ }^{1,2,3,4}$, Albrecht Berg 5 , Jürgen Popp ${ }^{1,2,3}$ and Karina Weber ${ }^{1,2,3 *}$

1) Friedrich Schiller University Jena, Institute of Physical Chemistry and Abbe Center of Photonics, Helmholtzweg 4, 07743 Jena, Germany

2) InfectoGnostics Research Campus Jena, Centre for Applied Research, Philosophenweg 7, 07743 Jena, Germany

3) Leibniz Institute of Photonic Technology - Member of the research alliance "Leibniz Health Technologies", Albert-Einstein-Str. 9, 07745 Jena, Germany

4) Abbott (Alere Technologies GmbH), Research and Development, Loebstedter Str. 103-105, 07749 Jena, Germany

5) INNOVENT e.V. Jena, Prüssingstraße 27 B, 07745 Jena, Germany

\section{$\underline{\text { Contents: }}$}

Figure S-1. Analytical agarose gel of the eight investigated respiratory species.

Figure S-2. Results of grey value analysis for samples preprocessed with expanded glass beads.

Figure S-3: Results of grey value analysis for samples preprocessed with PEI-modified PE particles.

Figure S-4: Results of grey value analysis for patient strain samples.

Figure S-5. Analytical agarose gel to monitor the amplification of S. pneumonia in a concentration range between $10^{2}$ and $10^{6} \mathrm{CFU} / \mathrm{ml}$.

Figure S-6. Photographic and SEM images of expanded glass beads and PEI-modified PE particles.

Figure S-7. Spotting layout for the capture probes.

*corresponding author: karina.weber@leibniz-ipht.de, phone: +49 3641206102

${ }^{\diamond}$ current address of S.H.: Jencam GmbH, Felsbachstraße 9, 07745 Jena, Germany

tSusanne Pahlow and Lydia Lehniger contributed equally to this work. 


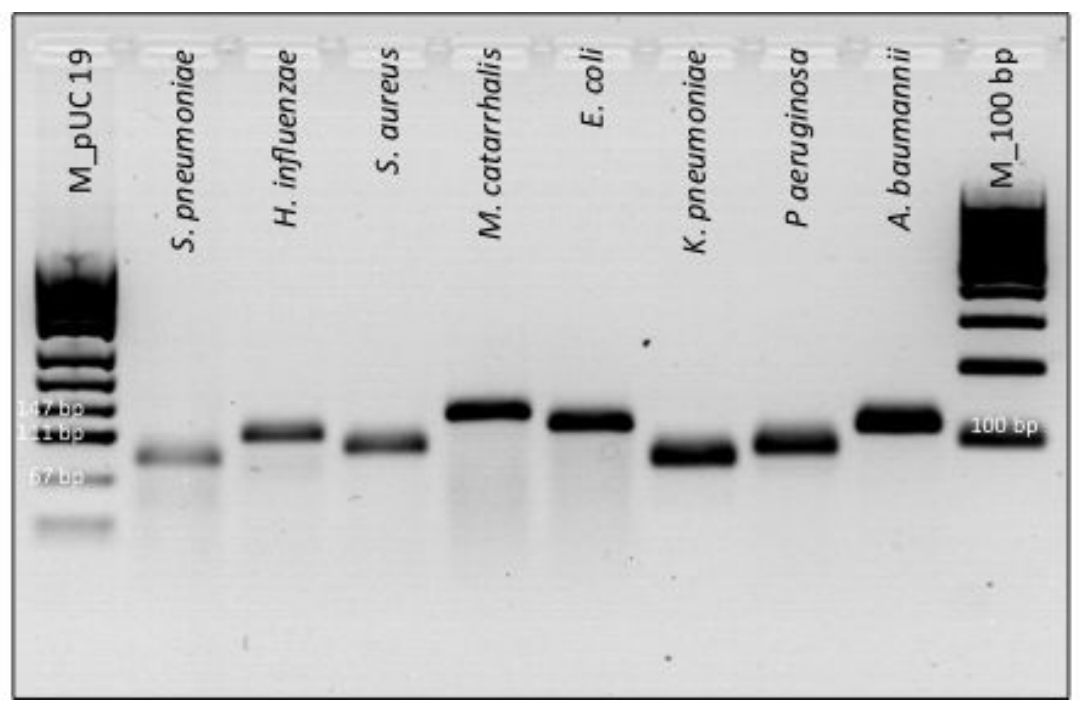

Figure S-1. Analytical agarose gel of the eight investigated respiratory species. 
Acinetobacter baumannii DSM 30007

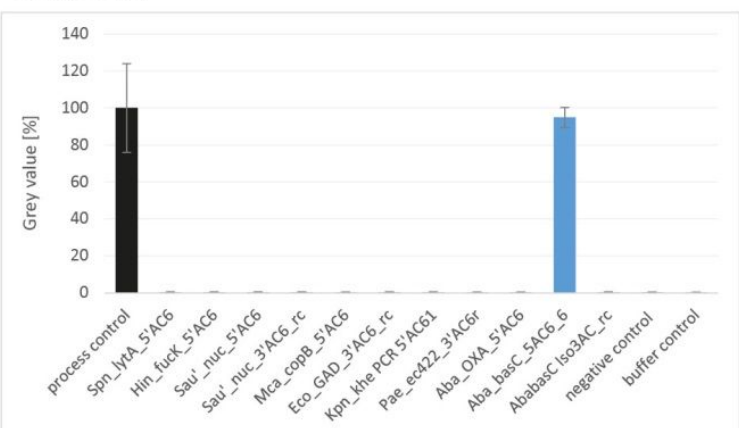

\section{Escherichia coli}

ATCC 25922

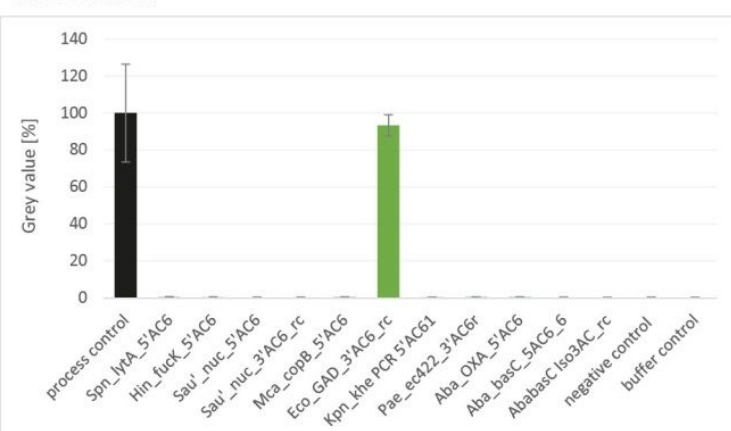

Haemophilus influenzae

ATCC 9007

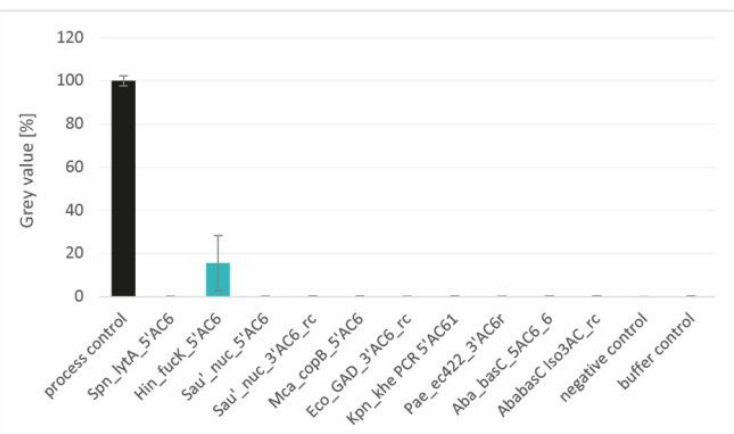

Staphylococcus aureus DSM 20231

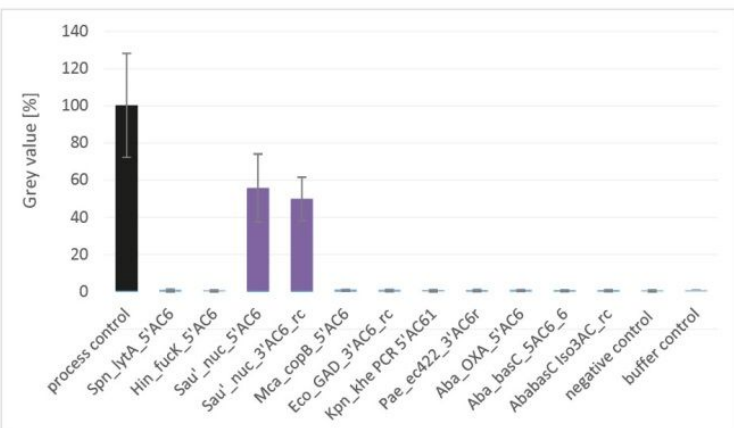

Klebsiella pneumoniae

DSM 30104

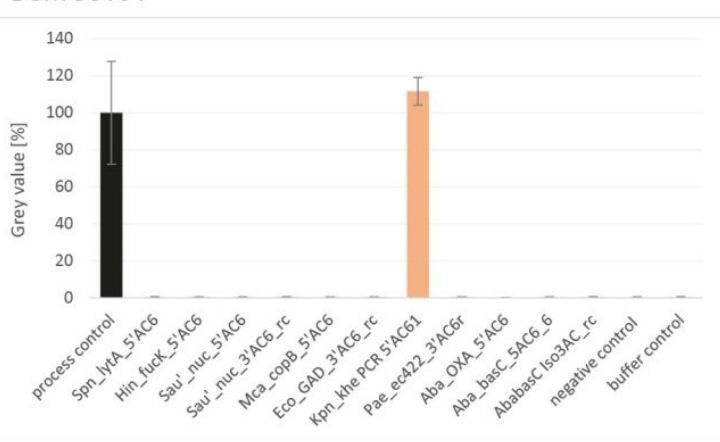

Moraxella catarrhalis

ATCC 43617

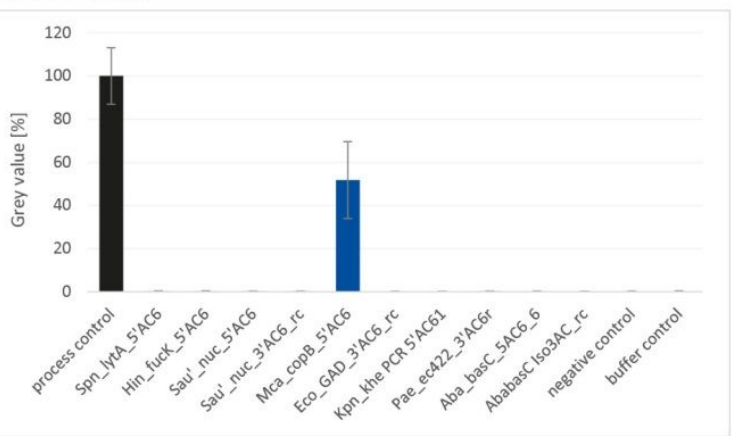

Pseudomonas aeruginosa

DSM 50071

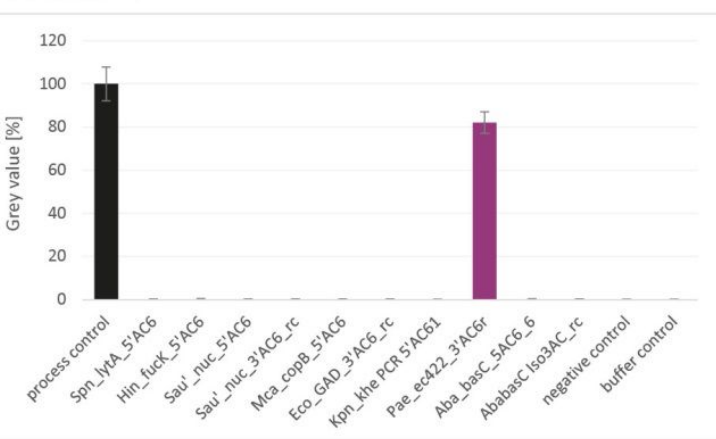

Streptococcus pneumoniae DSM 20566

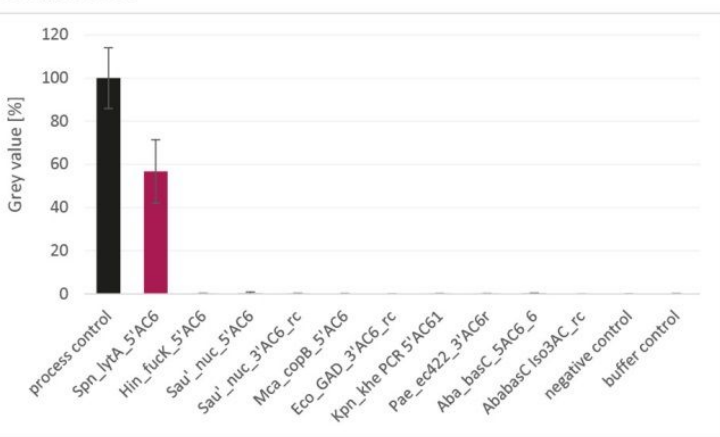

Figure S-2: Results of grey value analysis for samples preprocessed with expanded glass beads. 
Acinetobacter baumannii

DSM 30007

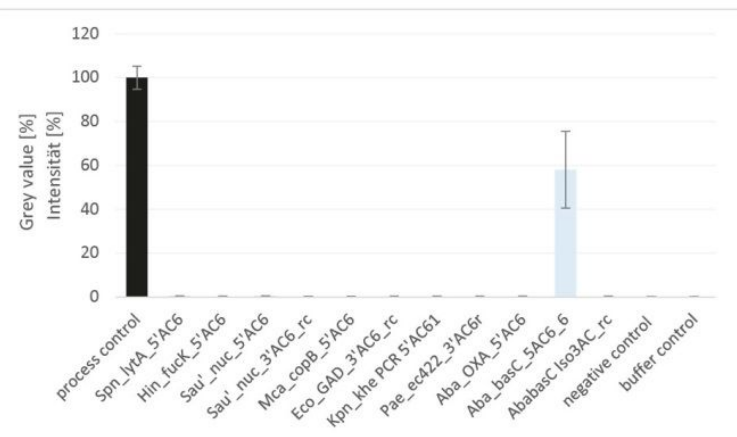

\section{Escherichia coli}

ATCC 25922

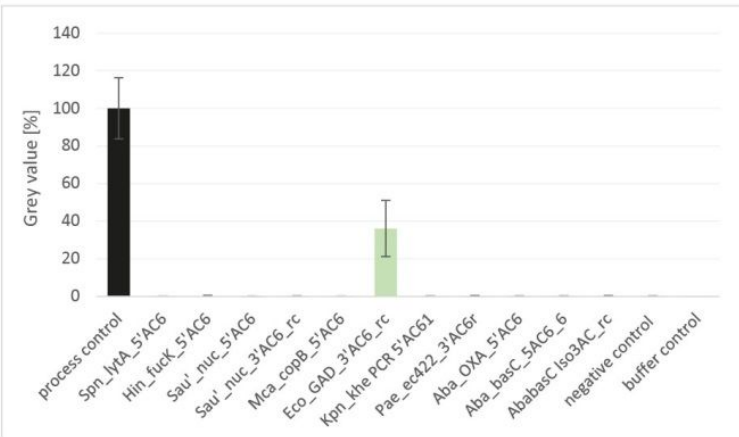

Haemophilus influenzae

ATCC 9007

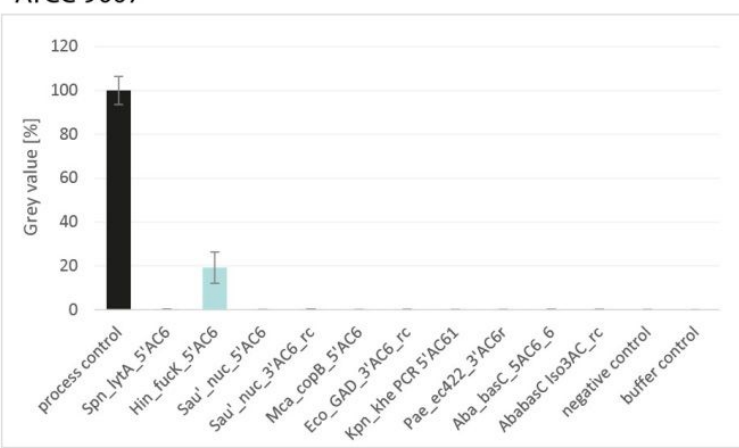

\section{Staphylococcus aureus} DSM 20231

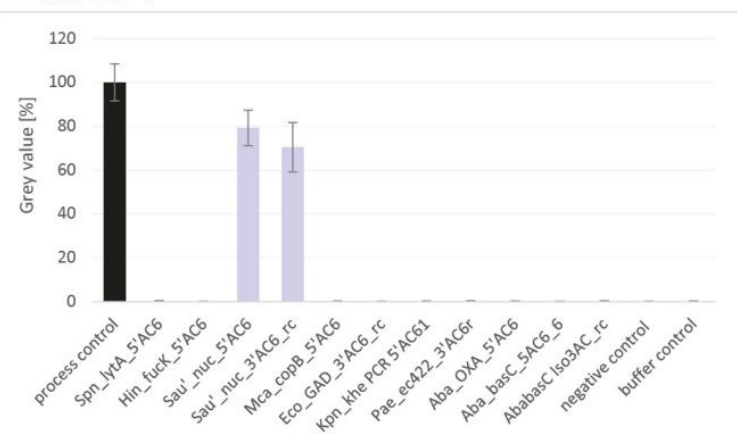

Klebsiella pneumoniae

DSM 30104

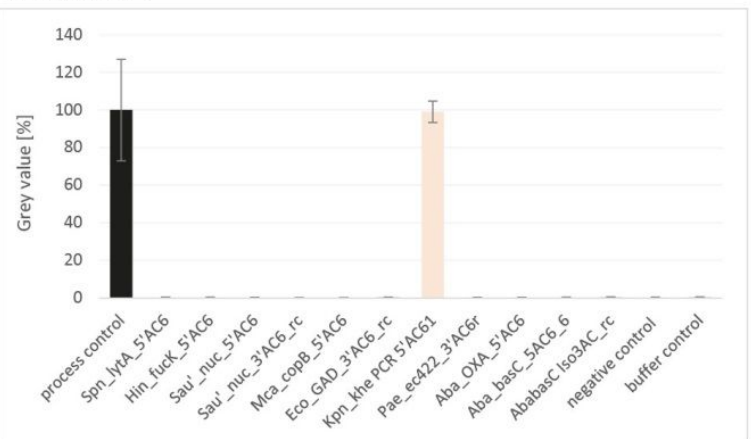

Moraxella catarrhalis

ATCC 43617

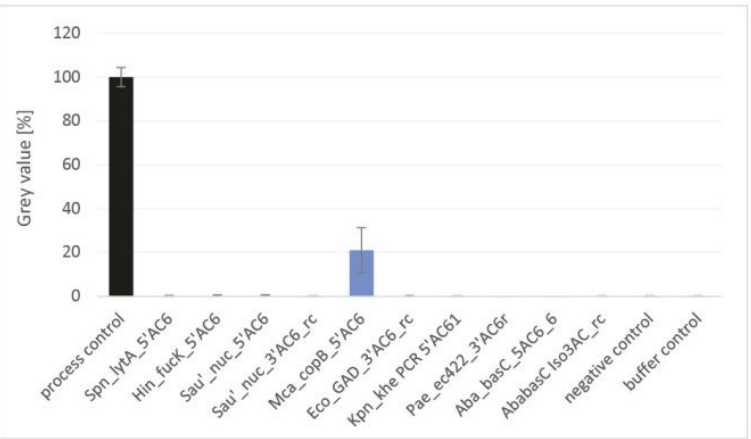

Pseudomonas aeruginosa

DSM 50071

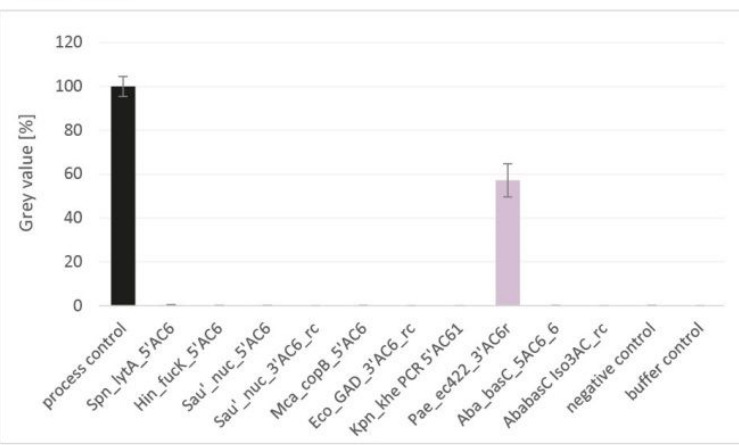

\section{Streptococcus pneumoniae}

DSM 20566

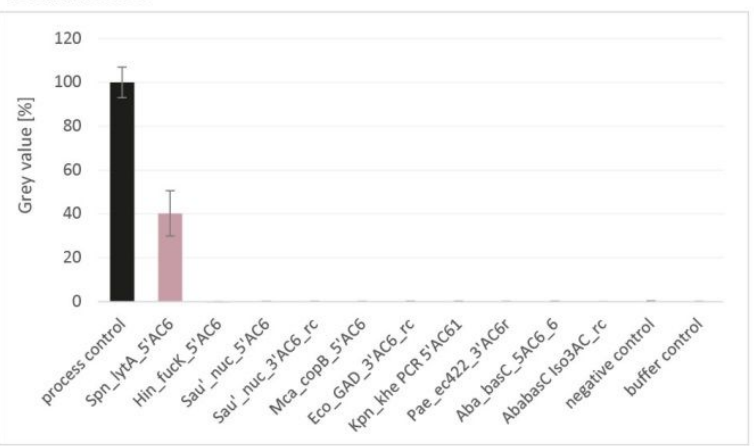

Figure S-3: Results of grey value analysis for samples preprocessed with PEI-modified PE particles. 
Haemophilus influenzae UK029

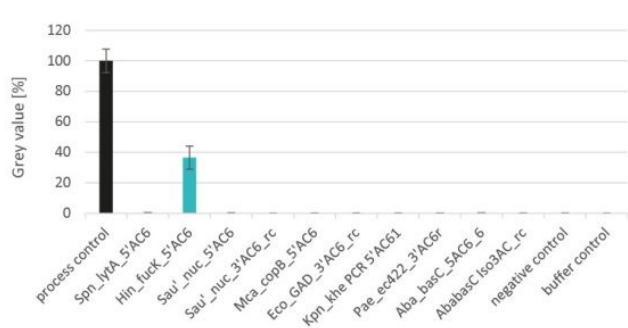

\section{Staphylococcus aureus}

UK001

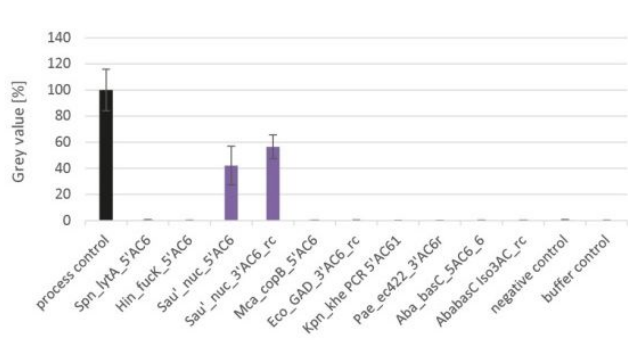

Staphylococcus aureus

UK017

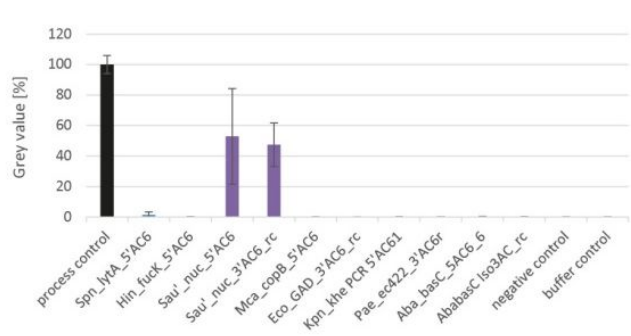

Staphylococcus aureus

UK019

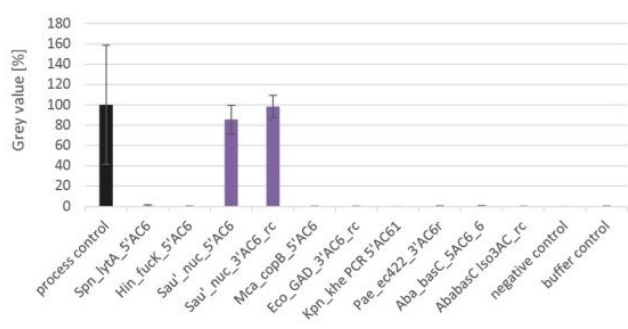

Streptococcus pneumoniae

UK033

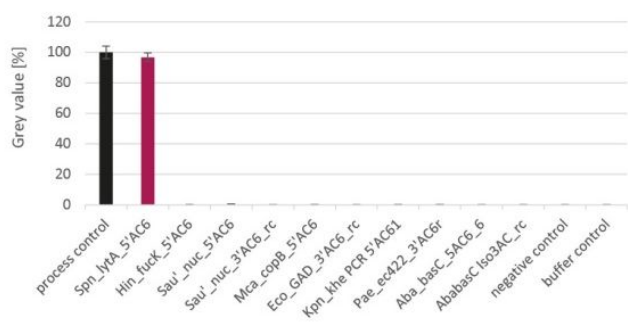

Haemophilus influenzae

UK030

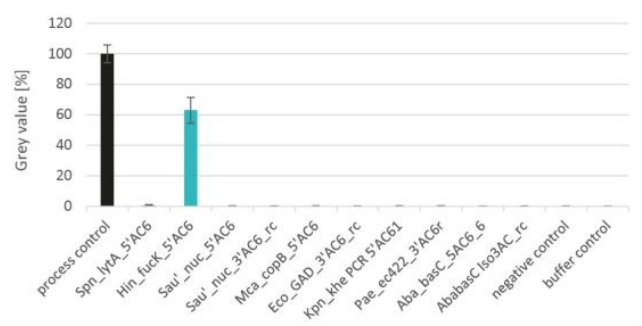

Staphylococcus aureus

UK002

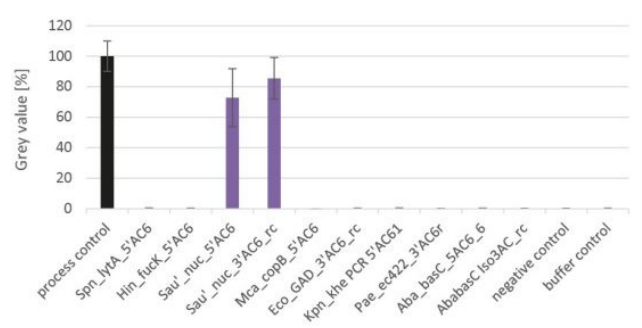

Staphylococcus aureus

UK018

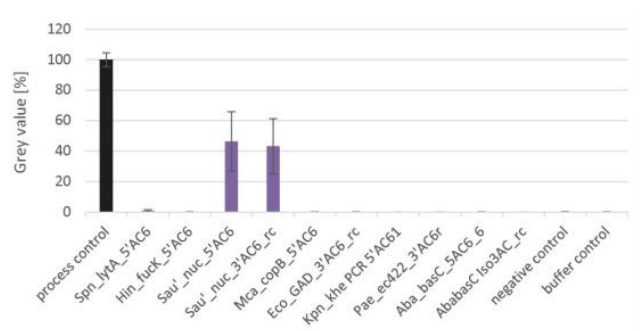

Streptococcus pneumoniae

UK032

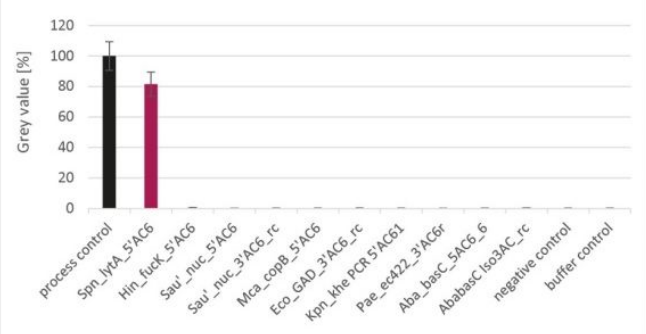

Streptococcus pneumoniae

UK034

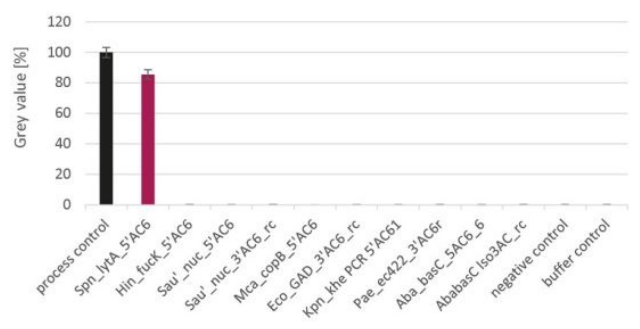

Figure S-4. Results of grey value analysis for patient strain samples. 


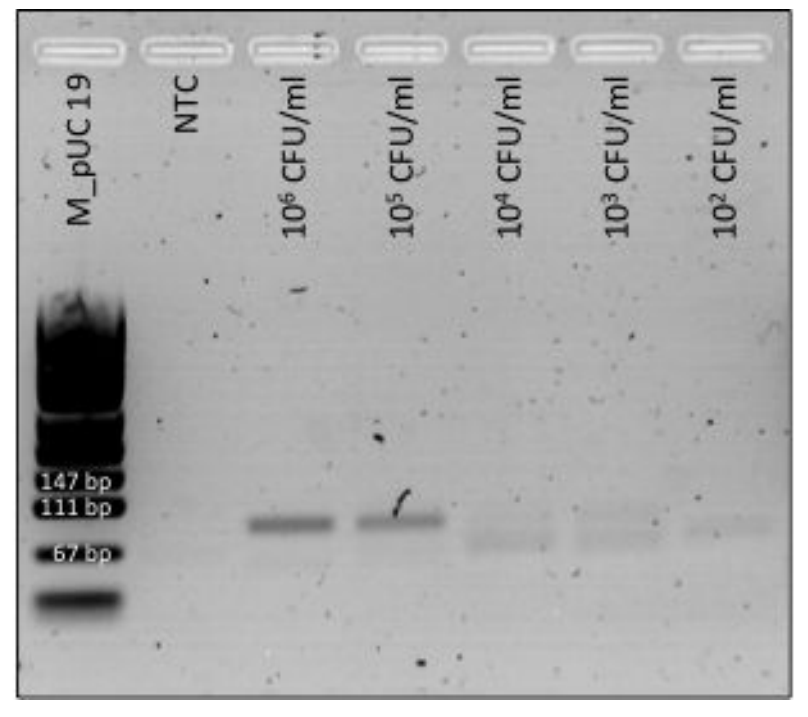

Figure S-5. Analytical agarose gel to monitor the amplification of $S$. pneumonia in a concentration range between $10^{2}$ and $10^{6} \mathrm{CFU} / \mathrm{ml}$. 

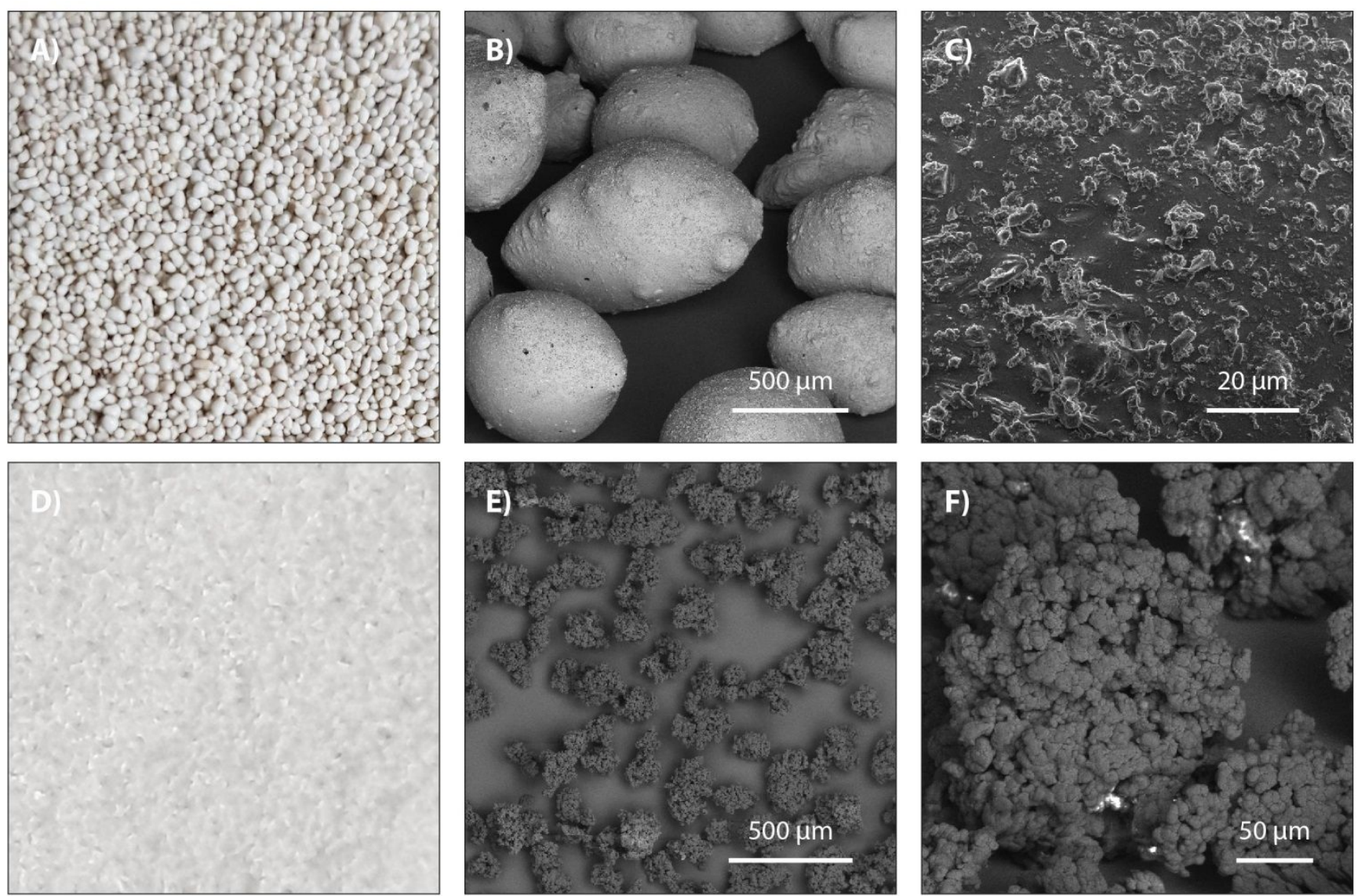

Figure S-6. A) Photographic images and B), C) SEM images of expanded glass beads, D) photographic images and E), F) SEM images of PEI-modified PE particles. 


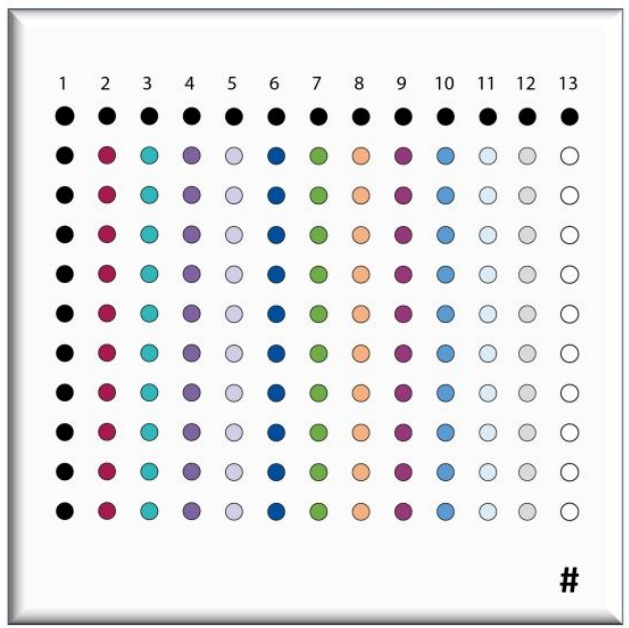

- 1 Process control

- 2 Spn_lytA_5'AC6

- 3 Hin_fuck_5'AC6

- 4 Sau_nuc_5'AC6

- 5 Sau_nuc_3'AC6_rc

6 Mca_copB_5'AC6

- 7 ECo_GAD_3'AC6_rC

O Kpn_khe PCR_5'AC61

- 9 Pae_ec422_3'AC6r

- 10 Aba_basC_5AC6_6

- 11 AbabasCIso3AC_rc

12 Negative contro

13 Spotting buffer

Figure S-7. Spotting layout for the capture probes. 